June 1997

revised June 1998

cond-mat/9806359

\title{
STATISTICAL MECHANICS OF VOTING
}

\author{
David A. Meyer* and Thad A. Brown ${ }^{\dagger}$ \\ Center for Social Computation and Institute for Physical Sciences \\ * Project in Geometry and Physics, Department of Mathematics \\ University of California/San Diego, La Jolla, CA 92093-0112 \\ dmeyer@chonji.ucsd.edu \\ ${ }^{\dagger}$ Department of Political Science, 113 Professional Building \\ University of Missouri/Columbia, Columbia, MO 65211 \\ polstab@showme.missouri.edu
}

\begin{abstract}
Decision procedures aggregating the preferences of multiple agents can produce cycles and hence outcomes which have been described heuristically as 'chaotic'. We make this description precise by constructing an explicit dynamical system from the agents' preferences and a voting rule. The dynamics form a one dimensional statistical mechanics model; this suggests the use of the topological entropy to quantify the complexity of the system. We formulate natural political/social questions about the expected complexity of a voting rule and degree of cohesion/diversity among agents in terms of random matrix models - ensembles of statistical mechanics models - and compute quantitative answers in some representative cases.
\end{abstract}

Journal of Economic Literature Classification System: D71, D72, C69.

1996 Physics and Astronomy Classification Scheme: 05.45.+b, 05.20.-y, 89.90.+n. American Mathematical Society Subject Classification: 90A28, 90A08, 54H20, 82B44.

Key Words: Condorcet cycle, Arrow's theorem, chaotic dynamical system, topological entropy, random matrix model, political cohesion, social diversity. 
The input for many mathematical models of social, political and economic systems includes a list of preference orders, one for each agent in the model. These preferences are aggregated, respectively, by some social welfare function, or voting rule, or market mechanism. More than 200 years ago, however, Condorcet recognized potential problems with voting rules, namely that aggregation might produce cycles [1]. For example, suppose that there are three alternatives $\{a, b, c\}$ and three voters rank them in the orders $a>b>c$ (by which we mean $a$ is preferred to $b$ which is preferred to $c$ ), $b>c>a$, and $c>a>b$. Given a choice between $b$ and $a$, a 2:1 majority prefers $a$; if they are offered the opportunity to switch from $a$ to $c$, again a majority will vote to do so; finally, a majority also prefers $b$ to $c$, completing a cycle.

While this example may seem contrived, Arrow's celebrated theorem [2] states that among an apparently reasonable set of voting rules, the only ones which do not encounter peculiarities of this sort for some profile (list of preference orders) are dictatorial, i.e., they depend only on the preference order of a single, specified, voter. Taking the example seriously then, we conceive it as describing a sequence of states (the successive preferred alternatives), a situation which is naturally modelled as a dynamical system. A similar perspective was originally suggested by Saari [3]; in this letter, motivated in part by potential applications to autonomous machines [4] choosing new states from a sequence of alternatives, rather than analyzing the situation by analogy with dynamical systems, we construct an explicit map from a profile and voting rule to a discrete dynamical system.

The usual model for a preference order is a relation, denoted $\geq$, which is complete (for all pairs of alternatives $a \geq b$ or $b \geq a$ ) and transitive (if $a \geq b$ and $b \geq c$ then $a \geq c$ ) [2]. When $a \geq b$ and $b \geq a$, the voter with this preference order is indifferent between $a$ and $b$; when only $a \geq b$, say, the voter strictly prefers $a$ and we write $a>b$. We consider aggregation formalized by maps $f$ from preference profiles $p$ to directed graphs $f_{p}$. A directed edge $a \leftarrow b$ in $f_{p}$ indicates that for profile $p$ the map $f$ chooses alternative $a$ over alternative $b$. We call $f$ a voting rule if
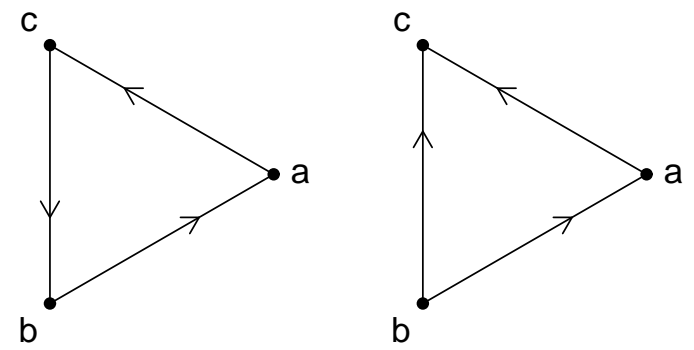
for all profiles $p, f_{p}$ is complete or a weak tournament [5] (for all pairs of alternatives $a \leftarrow b$ or $b \leftarrow a$ in $f_{p}$ ) and Pareto or unanimous (if $a \geq b$ in each preference order in $p$ then $a \leftarrow b$ in $f_{p}$ ). Notice that for every alternative $x$, since $x \geq x$ in every preference order, $x \leftarrow x$ in $f_{p}$ for every profile and voting rule. The weak tournament for the profile and majority voting rule of the example in the first paragraph is shown on the left in Fig. 1; we omit the edges connecting each vertex to itself.

We have motivated the introduction of weak tournaments by an example of what is essentially an amendment procedure [6], i.e., successive pairwise votes between a new 
alternative and the current one. Notice that this definition of voting rule is actually a generalization of the more familiar one which requires the outcome to be a preference order on the set of alternatives. Such an outcome corresponds to $f_{p}$ being transitive as well as complete and Pareto. But Arrow's theorem [2], for example, says that a broader definition is necessary if we forbid dictatorial rules and impose the condition of independence of irrelevant alternatives (IIA) - that the relation between $a$ and $b$ in $f_{p}$ depend only on the relations of $a$ and $b$ in the preference orders in $p$ [7]. In fact, the broader definition applies equally well to voters whose pairwise preferences are not necessarily consistent, i.e., transitive.

The directed graph which is the image of a voting rule $f$ on a specific profile $p$ defines a symbolic dynamical system: Suppose the voters are presented with a sequence of alternatives - an agenda, by extension of the usual meaning to allow arbitrarily long sequences. The results of successive pairwise votes between the new alternative and the current one form a sequence of symbols representing the chosen alternatives. The possible sequences are exactly the directed paths in $f_{p}$, e.g., for the first example in Fig. 1, baacbbaccc... are the first 10 symbols of an admissible sequence/path in $f_{p_{1}}$. For contrast, examine the second example in Fig. 1, obtained by applying the same majority voting rule to the profile $p_{2}=(a>b>c, c>b>a, c>a>b)$. An admissible sequence/path in $f_{p_{2}}$ can start the same way: baacccccc..., but once alternative $c$ is chosen, no other alternative can beat it; the sequence terminates with a string of $c$ s. It is clear that the space of admissible paths on $f_{p}$ completely characterizes a profile/voting rule pair. This space, together with the shift map (deletion of the first symbol of a sequence) forms the promised dynamical system - a (one-sided) subshift of finite type [8].

In Fig. 1, the first set of admissible sequences seems more interesting/complex than the second. To quantify this perception we enumerate the admissible sequences which are periodic with period $N$ : Define the transition matrix $F_{p}$ by $\left(F_{p}\right)_{a b}=1$ if $a \leftarrow b$ in $f_{p}$ and $\left(F_{p}\right)_{a b}=0$ otherwise. Then the number of $N$-periodic sequences is the trace of $F_{p}^{N}$. It is easy to check, for example, that $6=\operatorname{Tr} F_{p_{1}}^{3}>\operatorname{Tr} F_{p_{2}}^{3}=3$; more generally $\operatorname{Tr} F_{p}^{N}=\lambda_{1}^{N}+\cdots+\lambda_{k}^{N}$ when there are $k$ alternatives and $\lambda_{i}$ are the eigenvalues of $F_{p}$.

The thermodynamic formalism [9] provides a physical description of symbolic dynamical systems. Observe that

$$
\operatorname{Tr} F_{p}^{N}=\lim _{T \rightarrow 0} \sum_{\sigma \in A^{N}} e^{-E_{f_{p}}(\sigma) / T}=: \lim _{T \rightarrow 0} Z_{N}\left[f_{p}, T\right]
$$

where $A$ is the set of alternatives and, with the convention that $\sigma_{N+1} \equiv \sigma_{1}$,

$$
E_{f_{p}}(\sigma):=\sum_{i=1}^{N} 1-\left(F_{p}\right)_{\sigma_{i+1} \sigma_{i}}
$$

$Z_{N}\left[f_{p}, T\right]$ is the partition function for a statistical mechanics model on the lattice $\mathbb{Z}_{N}$ where the set of states is $A$ and the energy $E_{f_{p}}(\sigma)$ of a configuration $\sigma \in A^{N}$ is the 
sum of contributions from adjacent states: 0 if $\sigma_{i+1} \leftarrow \sigma_{i}$ in $f_{p}$ and 1 otherwise. The zero temperature $T \rightarrow 0$ limit in Eq. 1 eliminates the contributions from all but the ground state configurations, so the number of ground states (and hence $Z_{N}\left[f_{p}, 0\right]$ ) is the same as the number of admissible $N$-periodic sequences. From this perspective it is particularly natural to consider the free energy density (the average energy per lattice site) or equivalently, the topological entropy [10]:

$$
S\left[f_{p}\right]:=\lim _{N \rightarrow \infty} \frac{1}{N} \log Z_{N}\left[f_{p}, 0\right]
$$

Inserting Eq. 1 into Eq. 3 we see that $S\left[f_{p}\right]=\log \Lambda_{f_{p}}$, where $\Lambda_{f_{p}}$ is the spectral radius of $F_{p}$, namely its largest eigenvalue.

The topological entropy measures the degree of mixing of the dynamical system defined by $f_{p}$. When the entropy is positive the dynamical system is chaotic and exhibits the familiar features of chaos: topological transitivity, sensitive dependence on initial conditions, and a dense set of periodic points [11]. For the examples of Fig. 1, we can compute $S\left[f_{p_{1}}\right]=1$ (using logarithms in base 2 ) and $S\left[f_{p_{2}}\right]=0$, which suggests that the presence of a cycle in $f_{p}$ makes the dynamical system chaotic. This is true in general:

Proposition. The dynamical system defined by a complete directed graph has positive topological entropy iff the graph contains a nontrivial cycle.

Proof. If the directed graph has no nontrivial cycle there is some ordering of the vertices for which the associated transition matrix is upper triangular. Hence all its eigenvalues are 1, so the topological entropy vanishes. Conversely, suppose there is a cycle of length $l>1$ in the directed graph. Considering only those paths which lie entirely on the cycle, at each vertex of the cycle such a path may stay there or continue to the next vertex. Starting from any vertex on the $l$-cycle, then, there are $2^{N}$ such paths of length $N$, which may require at most $l-1$ additional steps to close. Thus $S \geq \lim _{N \rightarrow \infty}\left(\log l 2^{N}\right) / N=1$.

Since Arrow's theorem [2] guarantees the existence of cycles for any nondictatorial IIA voting rule and some profile on at least three alternatives, positivity of the topological entropy demonstrates the connection between Arrow's theorem, cycles and chaos hinted at by the 'chaos' theorems in spatial voting models [12] as well as by Saari's suggestive analogies [3]. We therefore propose to use the topological entropy as a measure of the complexity of a profile/voting rule pair. It identifies the associated dynamical system as chaotic or nonchaotic and quantifies 'how chaotic' the system is. Consider the pair of profiles $p_{3}=(b>c>a>d, c>d>a>b, d>b>c>a)$ and $p_{4}=(b>c>a>$
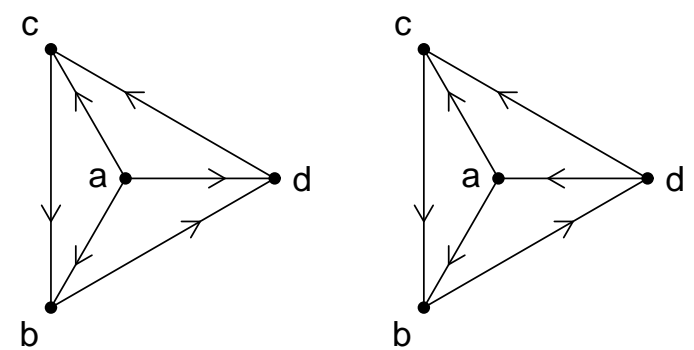

Fig. 2. The weak tournaments corresponding to majority rule on the profiles $p_{3}=(b>c>a>$ $d, c>d>a>b, d>b>c>a)$ and $p_{4}=(b>$ $c>a>d, c>a>d>b, d>b>c>a)$. On the left, $f_{p_{3}}$ contains a 3 -cycle; on the right, $f_{p_{4}}$ contains a 4-cycle. 
$d, c>a>d>b, d>b>c>a)$ for three voters and four alternatives. Majority rule gives the weak tournaments shown in Fig. 2, both of which contain cycles and so define chaotic dynamical systems. Notice that $f_{p_{3}}$ contains a 3 -cycle while $f_{p_{4}}$ contains a 4-cycle; the entropies differ correspondingly [13]:

$$
S\left[f_{p_{3}}\right]=1 \quad \text { and } \quad S\left[f_{p_{4}}\right] \approx 1.260
$$

Formulating the system as a statistical mechanics model focusses our attention on the energy functional: The energy of a configuration is defined (Eq. 2) by a matrix with entries $1-\left(F_{p}\right)_{a b}$. As the profile $p$ or the voting rule $f$ changes, this matrix can change. For a (probabilistic) ensemble of possible matrices the partition function (Eq. 1) defines a random matrix model [14].

The first type of ensemble we consider is generated by a random distribution of profiles. For a given voting rule, the statistical mechanics model defined by $Z_{N}\left[f_{p}, T\right]$ will be present in the ensemble with probability proportional to the number of profiles with the same image under $f$. For example, again consider the situation of majority voting on three alternatives. There are $(3 !)^{3}$ profiles for three voters with strict preferences, out of which $2 \cdot 3$ ! map to a weak tournament with a cycle as in $f_{p_{1}}$. Thus for a random ensemble over these profiles, the average entropy for majority rule is $1 \cdot \frac{1}{18}+0 \cdot \frac{17}{18}=\frac{1}{18} \approx 0.056$. In the limit of an infinite (odd) number of voters, we can use a result originally obtained by Guilbaud [15], that the probability of a cycle is $\frac{1}{4}-\frac{3}{2 \pi} \arcsin \frac{1}{3}$, to find that the average entropy for majority rule on three alternatives goes up to approximately 0.088 .

Similarly, for four alternatives, there are $(4 !)^{3}$ strict profiles for three voters, out of which 1632 map to a weak tournament with a 3 -cycle as in $f_{p_{3}}$ and 720 map to a weak tournament with a 4 -cycle as in $f_{p_{4}}$. Weighting the entropies in Eq. 4 accordingly, over this ensemble of profiles the average entropy for majority rule is approximately 0.184 . In the limit of an infinite (odd) number of voters, we can use a result of May and of Fishburn [16] that the probability of a Condorcet winner [13] is $\frac{1}{2}+\frac{3}{\pi} \arcsin \frac{1}{3}$, together with the result of Gehrlein and Fishburn [17] that the probability that there is no nontrivial cycle is

$$
\frac{3}{8}+\frac{6}{\pi^{2}} \int_{0}^{1 / 3} \frac{\arccos \left[-x /\left(1-2 x^{2}\right)\right]}{\left(1-x^{2}\right)^{1 / 2}} \mathrm{~d} x,
$$

to find that the average entropy for majority rule on four alternatives goes up to approximately 0.391 .

We can also consider the same ensembles of profiles aggregated by other voting rules. The Borda count [18], for example, assigns weights of $n-1, n-2, \ldots, 0$ to each voter's first, second, ..., last preferences, respectively, sums the weights of each alternative, and ranks the $n$ alternatives accordingly. Since there is now the possibility of alternatives with equal ranks, even though the resulting weak tournament is transitive, it may still contain cycles [19]. For three voters the average entropy for the Borda count on three alternatives is $\log 3 \cdot \frac{1}{18}+1 \cdot \frac{1}{6} \approx 0.255$, while on four alternatives it is $\log 3 \cdot \frac{1}{18}+1 \cdot \frac{37}{96} \approx 0.473$. 
For comparison, we may use the Copeland method [20] to make the weak tournaments obtained by majority rule transitive: assign each alternative a weight which is the number of incoming minus the number of outgoing edges and rank the alternatives accordingly. For three voters the average entropy of the Copeland method on three alternatives is $\log 3 \cdot \frac{1}{18} \approx 0.088$, while on four alternatives it is $\log 3 \cdot \frac{17}{144}+1 \cdot \frac{5}{96} \approx 0.239$, each of which, although higher than for majority rule, is lower than the corresponding average entropy for the Borda count.

The second type of ensemble is generated by a random distribution of voting rules. We consider, for example, a uniform distribution of rules which satisfy IIA and have image in the set of strict tournaments. (Strict tournaments are weak tournaments with exactly one edge between every pair of vertices.) Each such voting rule is defined by its images on the profiles restricted to all pairs of alternatives. For a pair of alternatives there are $2^{n}$ possibilities for the restriction of a profile of $n$ strict preferences. The voting rule maps each of these to an edge directed one of two ways between these alternatives in a strict tournament. Since voting rules are Pareto, the two unanimous restricted profiles have fixed images, but the remaining $2^{n}-2$ may be mapped, independently, to either directed edge. Thus, if there are $k$ alternatives, there are $2^{\left(2^{n}-2\right)\left(\begin{array}{c}k \\ 2\end{array}\right)}$ possible IIA voting rules for $n$ voters. Although this forms a huge ensemble of maps $f$, given a profile $p$, it is straighforward to determine with what probability each statistical mechanics model $Z_{N}\left[f_{p}, T\right]$ occurs in the ensemble.

For example, consider the profile $p_{5}=(c>a>b>d, d>a>c>b, a>c>d>b)$. Restricted to $\{a, b\}$ or to $\{b, c\}$ this profile is unanimous, so every voting rule, being Pareto, must map $p_{5}$ to a strict tournament with the edges $a \leftarrow b$ and $c \leftarrow b$. The other pairwise restrictions, however, are not unanimous. Since we are considering an ensemble of IIA voting rules, this means that $a \leftarrow c$ (or $c \leftarrow a$ ), $a \leftarrow d$ (or $d \leftarrow a$ ), $b \leftarrow d$ (or $d \leftarrow b$ ), and $c \leftarrow d$ (or $d \leftarrow c$ ) are independent events. Furthermore, since the voting rules in this ensemble map profiles to strict tournaments, each of the 16 resulting possibilities has probability $\frac{1}{16}$. No further analysis of the ensemble is necessary; we can immediately observe that with with probability $\frac{3}{16}$ the strict tournament to which $p_{5}$ maps has a 4cycle (like $f_{p_{4}}$ ), with probability $\frac{5}{16}$ it has a 3 -cycle (like $f_{p_{3}}$ ), and with probability $\frac{1}{2}$ it is transitive. Thus, using the entropies in Eq. 4 , the average entropy for $p_{5}$ over the ensemble of strict IIA voting rules is approximately $1.260 \cdot \frac{3}{16}+1 \cdot \frac{5}{16} \approx 0.549$. We can compare this to the average entropy for $p_{3}$ or $p_{4}$ over the same ensemble. Each of these profiles is only unanimous upon restriction to $\{a, c\}$; the consequent probabilities for a 4-cycle, a 3 -cycle, and transitivity are $\frac{9}{32}, \frac{11}{32}$, and $\frac{3}{8}$, respectively, leading to a larger average entropy of approximately 0.698 .

This shows that the average entropy over an ensemble of voting rules is a plausible measure of the cohesion or diversity in a society [21], as described by a profile: When the number of pairs on which the profile is unanimous decreases, the average entropy increases. Furthermore, it is sensitive to which pairs the voters rank consistently. It is clear that for this IIA ensemble, unanimity on two disjoint pairs, e.g., $\{a, c\}$ and $\{b, d\}$, does not reduce the entropy from the value found for $p_{3}$ and $p_{4}$. 
Taking a statistical mechanics approach to a general problem in social dynamics $[1,2,6,12,21,22]$ — iterated preference aggregation — we have been led to the topological entropy as a quantitative measure of the complexity of profile/voting rule pairs. Unlike traditional approaches which have concentrated merely on the existence (or not) of cycles, use of this quantitative measure allows comparison between systems differing even in number of voters or alternatives. Furthermore, we have constructed an annealed random matrix model for voting and considered ensembles corresponding to two natural social/political questions: What level of complexity can we expect from a given voting rule? How cohesive/diverse is the system relative to some collection of voting rules? The first question has been addressed in previous work by evaluating the probabilities for the existence of a Condorcet winner or of a nontrivial cycle $[15,16,17,23]$. Such probabilities are inputs into our calculations of the average entropy for a given ensemble of profiles and voting rule. Our approach to the second question is a strong generalization of the usual analysis of the (non)existence of a cycle for a single voting rule. Not only does the topological entropy provide for quantitative answers to these questions, it also makes precise the connection between the existence of cycles and chaos. Increasing the number of autonomous agents or the number of alternatives increases the complexity of the system; chaos can be reduced or avoided only by changing [23] or severely restricting [2,6,21] the class of agent preferences and/or aggregation rules.

\section{Acknowledgements}

We gratefully acknowledge support from the John Deere Foundation and from DISA, and useful discussions with Mike Freedman, Des Johnston, Jean-Pierre Meyer and Melanie Quong. 


\section{References and Notes}

[1] M. J. A. N. de Caritat, Marquis de Condorcet, Essai sur l'application de l'analyse à la probabilité des décisions rendues à la pluralité des voix (Paris: l'Imprimèrie Royale $1785)$.

[2] K. J. Arrow, Social Choice and Individual Values (New York: Wiley 1951);

For proofs of Arrow's theorem from a perspective congenial to physicists, see

G. Chichilnisky, "The topological equivalence of the Pareto condition and the existence of a dictator", J. Math. Econom. 9 (1982) 223-233;

Y. M. Baryshnikov, "Unifying impossibility theorems: a topological approach", Advances in Appl. Math. 14 (1993) 404-415.

[3] D. G. Saari, "The ultimate of chaos resulting from weighted voting systems", Advances in Appl. Math. 5 (1984) 286-308.

[4] B. Hasslacher and M. W. Tilden, "Living machines", Robotics and Autonomous Systems 15 (1995) 143-169.

[5] The adjective in 'weak tournament' allows, in analogy with the 'weak order' formalization of preference, the possibility that $f_{p}$ contain both $a \leftarrow b$ and $b \leftarrow a$. That is, both the individual preference orders in $p$ and their aggregate $f_{p}$ can include indifference between alternatives. For an early use of directed graphs/tournaments to analyze voting, see D. C. McGarvey, "A theorem on the construction of voting paradoxes", Econometrica 21 (1953) 608-610.

[6] D. Black, "On the rationale of group decision-making", J. Pol. Econom. 56 (1948) 23-34;

R. Farquharson, Theory of Voting (New Haven: Yale University Press 1969).

[7] This condition was earlier called the postulate of relevancy in E. V. Huntington, "A paradox in the scoring of competing teams", Science 88 (1938) 287-288.

[8] W. H. Gottshalk and G. A. Hedlund, Topological Dynamics, AMS colloquium publications, vol. 36 (Providence, RI: AMS 1955).

[9] D. Ruelle, Thermodynamic Formalism: The mathematical structures of classical equilibrium statistical mechanics (Reading, MA: Addison-Wesley 1978).

[10] C. E. Shannon, "A mathematical theory of communication", Bell System Tech. J. 27 (1948) 379-423; 623-656;

W. Parry, "Intrinsic Markov chains", Trans. Amer. Math. Soc. 112 (1964) 55-66;

R. L. Adler, A. G. Konheim and M. H. McAndrew, "Topological entropy", Trans. Amer. Math. Soc. 114 (1965) 309-319.

[11] T. Li and J. Yorke, "Period three implies chaos", Amer. Math. Monthly 82 (1975) 985-992;

M. Misiurewicz, "Horseshoes for continuous mappings of an interval", in C. Marchioro, ed., Dynamical Systems, proceedings of the CIME session, Bressanone, Italy, 19-27 June 1978 (Napoli, Italy: Liguori Editore 1980) 125-135.

[12] B. Ward, "Majority rule and allocation", J. Conflict Resolution 5 (1961) 379-389;

R. D. McKelvey, "Intransitivities in multidimensional voting models and some implications for agenda control", J. Econom. Theory 12 (1976) 472-482;

N. Schofield, "Instabilities of simple dynamic games", Rev. Econom. Stud. 40 (1978) $575-594$; 
L. Cohen, "Cyclic sets in multidimensional voting models", J. Econom. Theory 20 (1979) 1-12;

R. D. McKelvey, "General conditions for global intransitivities in formal voting models", Econometrica 47 (1979) 1085-1112;

E. C. Browne, P. A. James and M. A. Miller, "Simulation of global and local intransitivities in a simple voting game under majority rule", Behav. Sci. 36 (1991) 148-156; D. Richards, "Intransitivities in multidimensional spatial voting: period three implies chaos", Soc. Choice Welf. 11 (1994) 109-119.

[13] We must also note, however, that the entropy does not distinguish between the cycle in $f_{p_{3}}$ being a top cycle and being a bottom cycle, as it would be were all the edges from $a$ reversed, making $a$ the Condorcet winner (a pairwise unbeatable alternative).

[14] E. P. Wigner, "Characteristic vectors of bordered matrices with infinite dimensions", Ann. Math. 62 (1955) 548-564;

M. L. Mehta and M. Gaudin, "On the density of eigenvalues of a random matrix", Nucl. Phys. 18 (1960) 420-427;

F. J. Dyson, "Statistical theory of the energy levels of complex systems. I, II, III", J. Math. Phys. 3 (1962) 140-175.

[15] G. Th. Guilbaud, "Les théories de l'intérêt général et le problème logique de l'aggrégation", Économie Appliquée 5 (1952) 501-584.

[16] R. M. May, "Some mathematical remarks on the paradox of voting", Behav. Sci. 16 (1971) 143-151;

P. C. Fishburn, "A proof of May's theorem $P(m, 4)=2 P(m, 3)$ ", Behav. Sci. 18 (1973) 212.

[17] W. V. Gehrlein and P. C. Fishburn, "Probabilities of election outcomes for large electorates", J. Econom. Theory 19 (1978) 38-49.

[18] J.-C. de Borda, "Mémoire sur les élections au scrutin", Mémoires de l'Académie Royale des Sciences (1781) 657-665.

[19] The Borda count is not an IIA voting rule, so Arrow's theorem [2] does not apply. Nevertheless, alternatives may be equally ranked and, if we define the dynamics by choosing an equally ranked new alternative over the status quo, the system will then contain cycles.

[20] A. H. Copeland, "A 'reasonable' social welfare function", mimeographed notes, University of Michigan seminar on applications of mathematics to the social sciences (November 1951);

referred to in L. A. Goodman, "On methods of amalgamation", in R. M. Thrall, C. H. Coombs and R. L. Davis, eds., Decision Processes (New York: Wiley 1954) 39-48.

[21] G. Chichilnisky, "Social diversity, arbitrage, and gains from trade: a unified perspective on resource allocation", Amer. Econom. Rev. 84 (1994) 427-434.

[22] D. A. Meyer, "Towards the global: complexity, topology and chaos in modelling, simulation and computation", chao-dyn/9710005, InterJournal Complex Systems, Article [123], http://dynamics.bu.edu/InterJournal/.

[23] B. Jones, B. Radcliff, C. Taber and R. Timpone, "Condorcet winners and the paradox of voting: probability calculations for weak preference orders", Amer. Pol. Sci. Rev. 89 (1995) 137-144. 